\title{
Harmonisierung zugunsten von Patienten und Forschern
}

\author{
Rainer Warth, Michelle Salathé, Annette Magnin, Simona Berardi Vilei, Georges Imbert, \\ Marie-Monique Stadelmann, Aurel Perren
}

Affiliation siehe Box "Arbeitsgruppe und Autoren»

\begin{abstract}
Die neuen Vorlagen stellen einen schweizweiten Konsens betreffend Inhalt und Umfang der Patienteneinwilligung für die Weiterverwendung von Daten und humanen Proben für Forschungszwecke dar. Vorgestellt werden die von der Arbeitsgruppe «Biobanken» erarbeiteten Vorlagen für die Aufklärung und Einwilligung zur Weiterverwendung von Material und Daten für Forschungszwecke und das Biobankenreglement.
\end{abstract}

\section{Harmonisation en faveur des patients et des chercheurs}

L'Académie Suisse des Sciences Médicales (ASSM) a publié en 2006 les directives médico-éthiques «Biobanques». En se basant sur ces lignes directrices, un groupe de travail conjoint de la Fondation biobank-suisse (BBS) et l'ASSM a élaboré et publié en 2010 des modèles pour un consentement général (information et consentement éclairé) et pour une réglementation des biobanques [1]. Les modèles présentaient le premier consensus dans toute la Suisse concernant le contenu et la portée du consentement du patient pour la réutilisation des données et des échantillons humains à des fins de recherche. Depuis 2014, la loi fédérale relative à la recherche sur l'être humain (LRH) définit également le cadre juridique de la recherche avec des matériaux biologiques humains. En 2013 Swissethics, I'organisation faîtière des commissions d'éthique suisses pour la recherche, a chargé un groupe de travail dirigé par le professeur A. Perren d'élaborer de modèles d'information et de consentement, qui devraient faciliter l'application de LRH pour les chercheurs. Les modèles d'information et de consentement pour la réutilisation du matériel et des données à des fins de recherche et le règlement de biobanque développés par le groupe de travail «Biobanques» sont présentés ci-dessous. Les modèles sont publiés en allemand, anglais, français et italien sur le site web de Swissethics (www.swissethics.ch); ils sont mis à jour régulièrement.

\section{Arbeitsgruppe und Autoren}

Dr. Rainer Warth, Stiftung biobank-suisse (seit Nov. 2014 bei Swiss Biobanking Platform) Lic. iur. Michelle Salathé, Schweizerische Akademie der Medizinischen Wissenschaften SAMW

Dipl. pharm. Annette Magnin, Geschäftsführerin, Swiss Clinical Trial Organisation SCTO Dr. Simona Berardi Vilei, Schweizer Arbeitsgemeinschaft für Klinische Krebsforschung Dr. Georges Imbert, Novartis Pharma AG

Marie-Monique Stadelmann, MSc. Medical Drugs, Roche AG

Prof. Dr. med. Aurel Perren, Institut für Pathologie, Universität Bern

\section{Hintergründe}

Wieso werden Biomaterial und Gesundheitsdaten für Forschungszwecke gesammelt? Gesundheitsbezogene Personendaten und biologisches Material (Gewebe, Blut, Urin u.a.) sind eine wichtige Grundlage für die biomedizinische Forschung. Biologisches Material kann im Rahmen der medizinischen Diagnostik in Pathologieinstituten (Gewebebanken) oder in der Labormedizin (Biobanken für Flüssigkeiten) anfallen. Ausserdem wird in klinischen Studien, in Kohortenstudien oder in epidemiologischen Studien biologisches Material für Analysezwecke gesammelt. Mit den neuen IT-Techniken und der immer rascheren und günstigeren Verfügbarkeit von DNA-Sequenzanalysetechniken gewinnt biologisches Material zunehmend an Bedeutung. Aber auch andere omics-Analyseverfahren, wie Proteomics, Metabolomics, sind auf richtig gesammeltes biologisches Material und saubere Daten angewiesen, um aus der Fülle von Informationen relevante Erkenntnisse erlangen zu können.

Was sind die gesetzlichen Rahmenbestimmungen in der Schweiz?

2010 wurde der Bundesverfassungsartikel 118b «Forschung am Menschen» angenommen, der den Bundesrat ermächtigt, die Forschung am Menschen umfassend zu regeln. Gestützt darauf hat der Bundesrat das Bundesgesetz über die Forschung am Menschen (Humanforschungsgesetz, HFG) und die dazugehörigen Verordnungen erlassen, die am 1. Januar 2014 in Kraft getreten sind. 
Artikel 118 b definiert die Kompetenzen des Bundes wie folgt: «Der Bund erlässt Vorschriften über die Forschung am Menschen, soweit der Schutz seiner Würde und seiner Persönlichkeit es erfordert. Er wahrt dabei die Forschungsfreiheit und trägt der Bedeutung der Forschung für Gesundheit und Gesellschaft Rechnung." Art. 118b hält zudem die bei Forschungsvorhaben zu beachtenden Grundsätze fest. Zu diesen gehört auch die Einwilligung: «Jedes Forschungsvorhaben setzt voraus, dass die teilnehmenden oder gemäss Gesetz berechtigten Personen nach hinreichender Aufklärung ihre Einwilligung erteilt haben. Das Gesetz kann Ausnahmen vorsehen. Eine Ablehnung ist in jedem Fall verbindlich.»

Weitere Information zum Artikel 118b, dem HFG und den Ausführungsverordnungen sind sowohl auf der Website von Swissethics als auch auf der Website der Koordinationsstelle Forschung am Menschen (KOFAM), die dem Bundesamt für Gesundheit angegliedert ist (www.kofam.ch; www.swissethics.ch [2, 3]), zu finden.

BOX 1: a) Erster Teil der Einwilligung für die Weiterverwendung von biologischem Material und personenbezogenen Daten. b) Erster Teil der Patienteneinwilligung für die Verwendung von Material, das durch zusätzliche Massnahmen gewonnen wird.

a) Ich willige hiermit ein, dass mein zu Diagnose- oder Behandlungszwecken entnommenes biologisches Material und die Angaben zu meiner Erkrankung für die Forschung weiterverwendet werden dürfen. Die in der <<Institution >> zuständigen, genau bezeichneten Personen verschlüsseln das biologische Material, bevor es für einzelne Forschungsprojekte verwendet wird. Das bedeutet, dass die an einem Forschungsprojekt beteiligten Personen nicht wissen, dass das biologische Material von mir stammt ...

b) Ich willige hiermit ein, das mir durch $<<$ Massnahme $>$ biologisches Material und Daten für die Forschung entnommen bzw. erhoben werden können. Die in der $<<$ Institution>> zuständigen, genau bezeichneten Personen verschlüsseln das biologische Material und die Daten, bevor sie für einzelne Forschungsprojekte verwendet werden. Das bedeutet, dass die an einem Forschungsprojekt beteiligten Personen nicht wissen, dass das biologische Material und die Daten von mir stammen ...

BOX 2: Zweiter Teil der Einwilligung.

Ich bestätige, dass

- $\quad$ ich zu dieser Einwilligungserklärung gehörende Aufklärung erhalten habe.

- ich hinreichend über die Weiterverwendung von biologischem Material und gesundheitsbezogenen Daten für die biomedizinische Forschung informiert wurde.

- ich die Möglichkeit hatte, Fragen zu stellen, und diese zu meiner Zufriedenheit beantwortet wurden.

- meine Einwilligung freiwillig ist und mir insbesondere keine Vor- oder Nachteile entstehen, wie ich mich auch entscheide.

- Ich weiss, dass ich als Spenderin/Spender das Recht habe, über Ergebnisse, die meine Gesundheit direkt betreffen, informiert zu werden. Ich habe zur Kenntnis genommen, dass ich nur dann informiert werde, wenn ich in dieser Einwilligungserklärung (siehe unten) angekreuzt habe, dass ich vom Recht auf Information Gebrauch machen möchte.

$\square \quad$ Ich möchte über Ergebnisse, die meine Gesundheit betreffen, informiert werden (sofern Kontaktaufnahme und Information möglich).

- Ich weiss, dass ich diese Einwilligung jederzeit widerrufen kann, ohne Angabe von Gründen.

\section{Arbeitsgruppe «Biobanken»}

Die Arbeitsgruppe war eine von 27 Arbeitsgruppen, die Swissethics 2013 eingesetzt hat, um schweizweite mit dem HFG konforme Vorlagen zu erarbeiten. Sie setzte sich aus Vertretern der Industrie, der Schweizer Arbeitsgemeinschaft für Klinische Krebsforschung (SAKK), der Schweizerischen Akademie der Medizinischen Wissenschaften (SAMW), der Swiss Clinical Trial Organisation (SCTO) und der Biobanken (Stiftung biobanksuisse) zusammen. Im Zeitraum von Juli 2013 bis Juli 2014 wurden in vier Sitzungen und drei Telefonkonferenzen die nachstehend aufgeführten Vorlagen ausgearbeitet. Diese wurden am 24. Januar 2014 im Rahmen des vierten SwissBIG-Treffens in Bern den Betreibern von Biobanken vorgestellt:

- Aufklärung und Einwilligung für die Weiterverwendung von biologischem Material und personenbezogenen Daten für Forschungszwecke (HFV Art. 6);

- Aufklärung und Einwilligung für die Verwendung von Material, das durch zusätzliche Massnahmen gewonnen wird (HFV Art. 8);

- Biobanken-Reglement.

\section{Was steht in den Vorlagen und wie können diese eingesetzt werden?}

Die Arbeitsgruppe «Biobanken» hat ihre Arbeit auf den BBS/SAMW-Vorlagen von 2010 aufgebaut [1] und diese an das HFG angepasst. Berücksichtigt wurden zudem praktische Aspekte, insbesondere wurde versucht, die Informationsbedürfnisse von betroffenen Personen, mehrheitlich Patienten, zu beachten und dies angemessen umzusetzen.

Die Einwilligung ist etwa eine A4-Seite lang und ist in zwei Teile gegliedert. Im ersten Teil wird beschrieben, wozu die Person einwilligt (Box 1). Im zweiten Teil werden die Rechte der Person aufgeführt, deren Kenntnisnahme sie bestätigt (Box 2). Die Einwilligung wird von der Person unterschrieben und bleibt im Besitz der Biobank. In der Aufklärung zur Einwilligung wird der Patient in Form von Fragen und Antworten ausführlich informiert (Box 3). Zusammen mit einer Kopie der Einwilligung wird dem Patient die Information überlassen.

Das Biobanken-Reglement ist eine Vorlage für die Biobankverantwortlichen, um ihre Organisation und Arbeitsweise transparent darzustellen. Das Reglement soll auch öffentlich einsehbar sein für die Patienten, Spender oder andere interessierte Personen.

Die Vorlagen sind für die Schweiz konzipiert und sollen dazu beitragen, dass an Schweizer Spitälern einheitliche Dokumente eingesetzt werden. Die Vorlagen erfüllen auch international geltende ethische Standards 
BOX 3: Gezeigt werden alle Fragen, die in der Aufklärung für die Patienteneinwilligung zur Weiterverwendung von biologischem Material und personenbezogenen Daten behandelt werden. Die Kommentare in der Klammer beziehen sich auf Unterschiede zu der Aufklärung für die Patienteneinwilligung zur Verwendung von Material, das durch zusätzliche Massnahmen gewonnen wird.

- Was bedeutet Ihre Einwilligung? (Art des Materials und der Daten sowie Methode werden angegeben)

- Wie wird das biologische Material entnommen? (Erklärung der Methode)

- Was beinhalten die gesundheitsbezogenen Daten? (Entfällt)

- Wie werden Ihr biologisches Material und Ihre gesundheitsbezogenen Daten geschützt?

- Was heisst verschlüsseln?

- Wie werden Ihr biologisches Material und Ihre gesundheitsbezogenen Daten für die Forschung verwendet?

- Was geschieht mit den Forschungsergebnissen, die Ihre Gesundheit betreffen?

- Was geschieht mit den Forschungsergebnissen von meinen Proben und gesundheitsbezogenen Daten?

- Werde ich finanziell beteiligt, falls die Forschungsergebnisse zu einem Produkt führen?

- Habe ich ein Recht auf Einsicht in meine Daten?

- Habe ich ein Recht auf Widerruf?

- Möchten Sie noch mehr wissen?

Korrespondenz:

Rainer Warth, PhD

Swiss Biobanking Platform

CHUV

Av. P.-Decker 5, NES/04/4006

CH-1011 Lausanne

Tél. 0213143728

rainer.warth[at]chuv.ch und sind deshalb auch in multinationalen Forschungsprojekten einsetzbar.

Die Arbeitsgruppe empfiehlt, die Vorlagen mit dem lokalen Datenschutzbeauftragten, der Ethikkommission, der Spitaldirektion und dem Rechtsdienst abzusprechen. So kann gewährleistet werden, dass die Dokumente im richtigen Kontext und mit möglichst wenig zusätzlichem Kostenaufwand eingesetzt werden können.

\section{Ausblick}

Swissethics hat mit Einsetzung der Arbeitsgruppe «Biobanken» eine schweizweite Harmonisierung angestrebt; dies zum Nutzen von Patienten, Forschenden, Betreibern von Biobanken und Ethikkommissionen. Die Arbeitsgruppe ist sich aber bewusst, dass die Vorlagen permanent angepasst werden müssen, um neue Entwicklungen und Erkenntnisse aus der Praxis zu berücksichtigen. Es ist daher wünschenswert, dass die Nutzer der Vorlagen (Biobankenbetreiber, Spitäler) ihre Verbesserungsvorschläge an Swiss- ethics übermitteln, so dass sie in die Überarbeitung einfliessen können.

Die Einführung der Vorlagen ist aufwendig und die Implementierung der notwendigen Arbeitsabläufe in den Spitälern setzt IT-Lösungen und personelle Ressourcen voraus. Aktuell sind die einzelnen Spitäler unterschiedlich weit und auch die Vorlagen werden unterschiedlich umgesetzt. Wichtig zum jetzigen Zeitpunkt wäre daher eine Diskussion zu folgenden Punkten:

- Wie kann die Harmonisierung weiter verbessert werden?

- Wo liegen die Probleme in der praktischen Umsetzung?

- Wie können zwischen den verschiedenen Akteuren (BAG, Swissethics etc.) verbindliche Absprachen erzielt werden?

Patienten und Spender haben einen Anspruch auf hinreichende Aufklärung. Dies ist ein wichtiger Faktor, um das Vertrauen der Bevölkerung in die biomedizinische Forschung zu stärken. Die vorgestellten Vorlagen sollen dies unterstützen und garantieren, sie bieten

\section{Es ist wünschenswert, dass die Nutzer} der Vorlagen ihre Verbesserungsvorschläge an Swissethics übermitteln, damit sie in die Überarbeitung einfliessen können.

jedoch keine Hilfestellungen für die organisatorische Umsetzung. Die Swiss Biobank Platform (SBP), Swissethics, die Schweizerische Akademie der Medizinischen Wissenschaften (SAMW), die Swiss Clinical Trial Organisation (SCTO) und die Schweizer Arbeitsgemeinschaft für Klinische Krebsforschung (SAKK) sind aufgefordert, die aufgeführten Diskussionspunkte aufzugreifen und Lösungsvorschläge zu erarbeiten.

\section{Referenzen}

1 Salathé M. Vorlagen für eine «Generaleinwilligung» und für ein Reglement. Schweiz Ärztezeitung. 2010;91(19/20):761-3.

2 www.kofam.ch (Stand 13.02.2015).

3 www.swissethics.ch/templates.html (Stand 13.02.2015), siehe $\rightarrow$ Studieninformationen und -einwilligungen, Biobankenreglement $\rightarrow$ Biobanken. 